\title{
РЕЗУЛЬТАТЫ ПРОКСИМАЛЬНОЙ РЕЗЕКЦИИ ЖЕЛУДКА В ЗАВИСИМОСТИ ОТ МЕТОДОВ РЕКОНСТРУКЦИИ ЖЕЛУДОЧНО-КИШЕЧНОГО ТРАКТА
}

\section{RESULTS OF THE PROXIMAL GASTRIC RESECTION DEPENDING ON METHODS OF GASTROINTESTINAL TRACT RECONSTRUCTION}

V. Tishakova

D. Ruchkin

A. Gritskevich

N. Efimenko

Summary. The article reflects the results of a study aimed at evaluating the effectiveness and prove the feasibility of using isoperistaltic jejunogastroplasty (modification of the Merendino-Dillard operation) as a method of primary reconstruction after subtotal proximal gastrectomy.

The analysis of the results of treatment of 97 patients operated on for surgical diseases of the cardiac stomach in the period from 2011 to 2019 was carried out. $92.8 \%$ of them had malignant neoplasms of the esophagogastric junction. Proximal gastric resection (PRG) was performed using isoperistaltic jejunogastroplasty (modified by Merendino-Dillard) (50 patients - Group I or main) and standard PRG with direct esophagogastroanastomosis (47 patients - Group II or control group).

The closest results in the main group compared with the control group did not have significant differences: blood loss was $410 \pm 16$ and $360 \pm 13$ $\mathrm{ml}$; the duration of stay (bed-days) in the ICU -0.9 days. and 2.2 days, in the hospital - 10.1 days. and 13.4 days. respectively. Complications developed in 25 patients (25.8\%): in group I there were 13 (26.0\%), in group II $-12(25.5 \%)$, while the frequency of complications associated with surgery (surgical) was $8(16.0 \%)$ and 7 cases (14.9\%), respectively ( $p>0.05$ ). Postoperative complications of the longterm period (after 12 months) associated with the reconstruction of the gastrointestinal tract were noted in 18 (20.9\%) patients: with a direct esophageal-gastric anastomosis — in $13(31.0 \%)$ versus $5(11.3 \%)$ during reconstruction of the gastrointestinal tract with jejunogastroplasty modified by Merendino-Dillard, while the frequency of reflux esophagitis was observed in group I - in $2(4.5 \%)$ and in group II - in $6(14.3 \%)$ cases $(p<0,05)$. The indicator of good health in the main group was $72.7 \%$, in the control $-47.8 \%(p<0.01)$.
Тишакова Виктория Эдуардовна

Врач-хирург, Центр хирургии филиала № 1 ФГБУ «Главный военный клинический госпиталь им. академика Н.Н. Бурденко» МО РФ (Москва); соискатель, ФГБУ «Национальный медицинский исследовательский центр хирургии им. А.В. Вишневского» Минздрава России tishakova21@gmail.com

Ручкин Дмитрий Валерьевич

Д.м.н., ФГБУ «Национальный медицинский исследовательский чентр хирургии им. А.В. Вишневского» Минздрава России (Москва) ruchkindmitry@gmail.com

Грицкевич Александр Анатольевич

Д.м.н., профессор, ФГБУ «Начиональный медицинский исследовательский чентр хирургии им. А.В. Вишневского» Минздрава России (Москва); Российский университет дружбы народов (Москва) vishnevskogo@ixv.ru

Ефименко Николай Алексеевич

Член-корреспондент РАН, д.м.н., профессор, хирург-консультант, Центр хирургии филиала № 1 ФГБУ «Главный военный клинический госпиталь им. академика Н.Н. Бурденко» МО РФ Москва) chiefsurgeon@mail.ru

Аннотация. В статье отражены результаты исследования, целью которых было оценить эффективность и доказать целесообразность применения изоперистальтической еюногастропластики (модификация операции Merendino-Dillard) в качестве способа первичной реконструкции после субтотальной проксимальной резекции желудка.

Проведен анализ результатов лечения 97 пациентов, оперированных по поводу хирургических заболеваний кардиального отдела желудка в период с 2011 по 2019 гг. Злокачественные новообразования пищеводно-желудочного перехода имели 92,8\% из них. Выполняли проксимальную резекцию желудка (ПРЖ) с применением изоперистальтической еюногастропластики (в модификации Merendino-Dillard) (50 больных — I-я группа или основная) и стандартную ПРЖ с прямым эзофагогастроанастомозом (47 больных - I-я группа или контрольная группа).

Ближайшие результаты в основной группе по сравнению с контрольной не имели достоверных различий: кровопотеря составила $410 \pm 16$

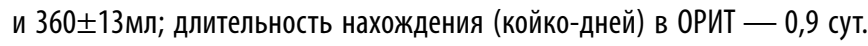
и 2,2 сут., в стационаре - 10,1сут. и 13,4 сут. соответственно. Осложнения развились у 25 пациентов (25,8\%): в І-й группе их было 13 (26,0\%), во II-й - 12 (25,5\%), при этом частота осложнений, связанных с опера- 
The safety and physiological effectiveness of isoperistaltic jejunogastroplasty (a modification of the Merendino-Dillard operation) make it the method of choice after subtotal proximal gastrectomy.

Keywords: stoma cancer, proximal resection of the stomach, reconstruction with proximal resection of the stomach. тивным вмешательством (хирургических) составила 8 (16,0\%) и 7 случаев $(14,9 \%)$ соответственно ( $>>0,05)$. Послеоперационные осложнения отдаленного периода (через 12 мес.), связанные с реконструкцией ЖКТ, отмечены у 18 (20,9\%) пациентов: при прямом пищеводно-желудочном анастомозе - у 13 (31,0\%) против 5 (11,3\%) при реконструкции ЖКТ еюногастропластикой в модификации Merendino-Dillard, при этом частота рефлюкс-эзофагита наблюдалась в I-й группе — в 2 (4,5\%) и во II-й — в 6 $(14,3 \%)$ случаях $(p<0,05)$. Показатель хорошего самочувствия пациентов основной группы составил $72,7 \%$, контрольной $-47,8 \%(p<0,01)$.

Безопасность и физиологическая эффективность изоперистальтической еюногастропластики (модификация операции Merendino-Dillard) позволяют считать её способом выбора после субтотальной проксимальной резекции желудка.

Ключевые слова: рак желудка, проксимальная резекция желудка, реконструкция при проксимальной резекции желудка.

\section{Шель исслеАования}

Цель настоящего исследования - доказать эффективность и целесообразность применения изоперистальтической еюногастропластики (модификация операции Merendino-Dillard) в качестве способа первичной реконструкции после субтотальной проксимальной резекции желудка.

\section{Материал и метомы}

Проведен сравнительный анализ результатов лечения 97 пациентов, оперированных в ФГБУ «НМИЦ хирургии им. А.В.Вишневского» Минздрава РФ (50 больных), а также филиале № 1 ФГБУ «ГВКГ им. Н.Н. Бурденко» Минобороны РФ и ФГКУ «ЦВКГ им. П.В. Мандрыка» МО РФ (47 больных) за период с 2011 по 2019 гг.

Выполняли проксимальную резекцию желудка (ПРЖ) с применением изоперистальтической еюногастропластики (в модификации Merendino-Dillard) (50 больных - І-я группа или основная) (рис. 1) и стандартную ПРЖ с прямым эзофагогастроанастомозом (47 больных - II-я группа или контрольная группа) (рис. 2).

В анализируемых группах было 61,9\% мужчин и 38,1\% женщин. Возраст пациентов варьировал в пределах от 48 до 77 лет. Средний возраст в І-й группе со-

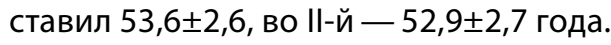

Рак пищеводно-желудочного перехода выявлен у 90 (92,8\%) пациентов; в І-й группе у 46 (92,0\%), во II-й - у 44 (93,6\%). Чаще всего оперативному лечению подвергались пациенты с ранним раком (рТ1a-b; $\left.\mathrm{T}_{1} \mathrm{~N}_{1-2} \mathrm{M}_{0}\right)-81$ (90\%): в І-й группе - 41 (89,1\%) пациент, во II-й - 40 $(90,9 \%)$ пациентов. 


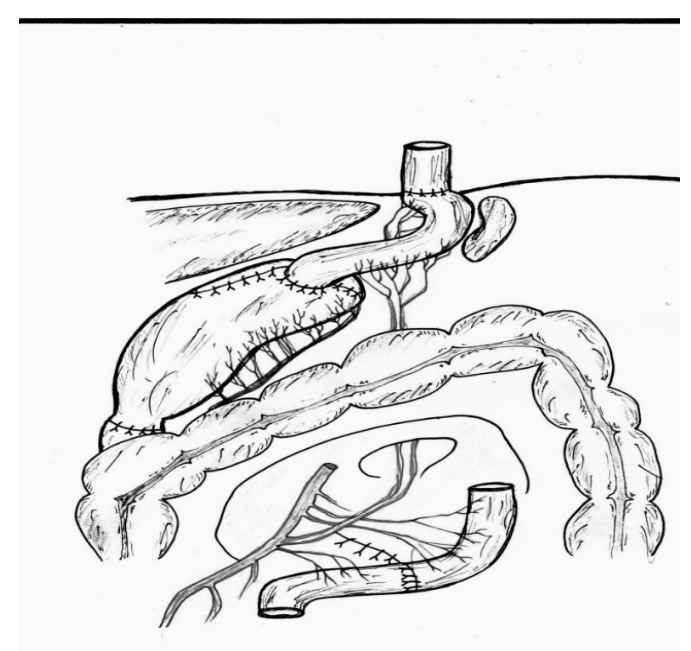

Рис. 1. Изоперистальтическая еюногастропластика в модификации Merendino-Dillard (схема)
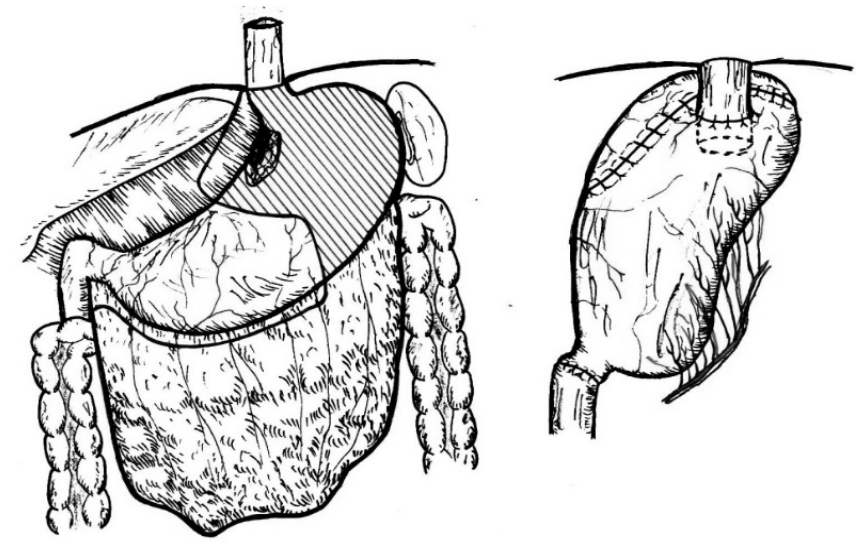

Рис. 2. Прямой пищеводно-желудочный анастомоз после ПРЖ (схема)

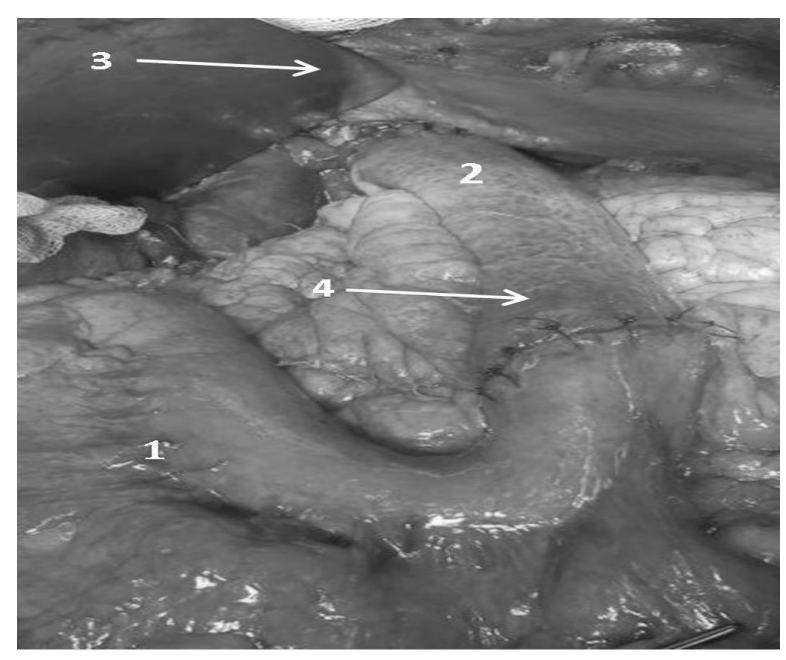

Pис. 3. Еюногастропластика в модификации Merendino-Dillard: 1 - желудочная трубка;

2 - изоперистальтический сегмент тонкой кишки; 3 - эзофагоеюноанастомоз; 4 гастроеюноанастомоз

По локорегионарной и отдаленной распространённости опухолевого процесса, а также по тяжести и частоте сопутствующих заболеваний в группах, статистически значимых различий не получено $(p>0,05)$.

Методика изоперистальтической еюногастропластики (модификация Merendino-Dillard) после субтотальной проксимальной резекции желудка

После мобилизации проксимального отдела желудка и абдоминального отдела пищевода, выполняли резекцию желудка и абдоминального отдела пищевода с использованием антирефлюксной вставки из петли тощей кишки на сосудистой ножке, между пищеводом и дистальной частью резецированного желудка.

При выборе кишечного трансплантата использовали начальную часть тощей кишки, начиная примерно на 15-20 см дистальнее связки Трейца с питанием на 3-ей или 4-й кишечной артерии. При формировании трансплантата, пересечение брыжейки и сосудистых дуг проводили всегда под контролем зрения, используя трансиллюминацию. После создания тонкокишечного сегмента его перемещали в верхний этаж брюшной полости через сформированное «окно» в мезоколон, не допуская осевого перекрута питающей ножки и со- 
Таблица 1. Непосредственные результаты проксимальной резекции желудка

\begin{tabular}{|l|l|l|l|} 
Критерии & $\begin{array}{l}\text { Гастропластика } \\
\text { в модификации Merendino- } \\
\text { Dillard } \\
\text { (l-я группа, } \mathbf{n = 5 0 )}\end{array}$ & $\begin{array}{l}\text { Прямой пищеводно- } \\
\text { желудочный анастомоз (II-я } \\
\text { группа, } \mathbf{n = 4 7 )}\end{array}$ & $\begin{array}{l}\text { Всего } \\
\text { (n=97) }\end{array}$ \\
\hline Продолжительность операции, мин & $318 \pm 11$ & $264 \pm 10$ & $291 \pm 10$ \\
\hline Объем кровопотери, мл & $410 \pm 16$ & $360 \pm 13$ & $385 \pm 14$ \\
\hline Койко-дни в ОРИТ, сутки & 0,9 & 2,2 & 1,6 \\
\hline Койко-дни в стационаре, сутки & 10,1 & 13,4 & 11,8 \\
\hline Частота осложнений, абс/\% & $13 / 26,0$ & $12 / 25,5$ & $25 / 25,8$ \\
\hline $\begin{array}{l}\text { Частота осложнений, связанных } \\
\text { соперацией, абс/\% }\end{array}$ & $8 / 16,0$ & $7 / 14,9$ & $15 / 15,5$ \\
\hline Летальность, абс/\% & - & $1 / 2,1$ & $1 / 1,0$ \\
\hline
\end{tabular}

храняя его изоперистальтическую направленность. Завершали ЕГП последовательным формированием трех дигестивных соустий:

1. межкишечного еюноеюноанастомоза;

2. еюногастроанастомоза;

3. эзофагоеюноанастомоза. Такая последовательность позволяла получить дополнительное время для оценки жизнеспособности уже перемещенного выше мезоколон трансплантата, заметить концевой некроз или гематому (рис. 3).

\section{Результаты}

Для оценки непосредственных результатов хирургических вмешательств использовали такие показатели, как длительность оперативного вмешательства, объём интраоперационной кровопотери, время нахождения в палате интенсивной терапии и в стационаре, частота послеоперационных осложнений и летальности (табл. 1).

Существенно значимой оказалась разница в группах лишь по продолжительности операции - более

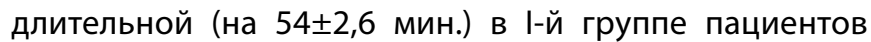
$(p<0,05)$. По остальным сравниваемым показателям результаты достоверных различий не имели ( $>>0,05)$.

Осложнения развились у 25 (25,8\%) пациентов: в І-й группе их было 13 (26,0\%), во II-й - 12 (25,5\%).

Частота общесоматических осложнений составила в общей популяции 10 (10,3\%) случаев: в І-й группе пневмония была в 4 случаях, пароксизмальная мерцательная аритмия - в 1; во II-й группе - пневмонии были в 3 случаях, нестабильная стенокардия у пациента с обострением хронической сердечной недостаточности - в 1, пароксизмальная мерцательная аритмия - в 1.

Что же касается осложнений, связанных с оперативным вмешательством, то они установлены у 15
(15,5\%) пациентов: в І-й группе - у 8 (16,0\%), во II-й у 7 (14,9\%). Из них наиболее клинически значимыми оказалась несостоятельность различных вариантов анастомозов, которая наблюдалась у 4 (4,1\%) пациентов: в І-й группе - у 2 (4,0\%) пациентов была выявлена несостоятельность пищеводно-кишечного анастомоза (трансплантата); во II-й группе - у 2 (4,3\%) - несостоятельность пищеводно-желудочного анастомоза.

Несостоятельность анастомозов была ликвидирована при использовании следующего лечебного алгоритма: адекватное дренирование зоны несостоятельности (в зависимости от уровня недостаточности) с помощью дренажа, установленного во время операции или под УЗ-контролем с последующей вакуум-аспирацией или проточно-аспирационным дренированием, проведение интенсивной терапии, обеспечение внутривенной и зондовой алиментарной поддержки. Частичные дефекты соустья зажили и пациентов выписали из стационара с возможностью полноценного питания через рот.

Послеоперационная летальность составила 1,03\%: после ПРЖ с «прямым» пищеводно-желудочным анастомозом (контрольная группа) умер 1 (2,12\%) пациент с нестабильной стенокардией, вследствие обострения хронической сердечной недостаточности, при этом на аутопсии каких-либо осложнений, связанных с оперативным лечением не выявлено. Летальных исходов среди пациентов основной группы не было.

В отдалённом периоде сроки наблюдения находились в интервале от 3 мес. до 3 лет, составив в среднем $28,3 \pm 2,2$ мес. в І-й группе пациентов и 27,2 $\pm 1,8$ мес. во II-й группе.

Через 12 мес. после операции нам удалось проследить за 44 пациентами из 1-й группы и за 42 из 2-й. Через 24 мес. и более - за 34 пациентами из 1-й группы и 30 из 2-й соответственно. 
Таблица 2. Характеристика питательного статуса после проксимальной резекции желудка, в зависимости от характера реконструкции желудочно-кишечного тракта (через 24 мес. и более, абс./\%)

\begin{tabular}{|c|c|c|c|}
\hline \multirow{2}{*}{ Критерии } & & \multicolumn{2}{|c|}{ Проксимальная резекция желудка } \\
\hline & & $\begin{array}{l}\text { Гастропластика } \\
\text { в модификации Merendino- } \\
\text { Dillard } \\
\text { (I-я группа, n=34) }\end{array}$ & $\begin{array}{l}\text { Прямой пищеводно-желудочный } \\
\text { анастомоз (II-я группа, n=30) }\end{array}$ \\
\hline \multirow{3}{*}{ Режим питания (раз в день) } & $3-4$ & $23 / 67,6$ & $11 / 36,7$ \\
\hline & $5-6$ & $9 / 26,5$ & $12 / 40,0$ \\
\hline & 7 и более & $2 / 5,9$ & $7 / 23,3$ \\
\hline \multirow{2}{*}{ Динамика массы тела } & При выписке & 90,7 & 89,7 \\
\hline & Через 24 мес. & 109,9 & 96,9 \\
\hline \multirow{2}{*}{ Частота стула в день } & $1-2$ & $30 / 88,2$ & $23 / 76,7$ \\
\hline & диарея & $4 / 11,8$ & $7 / 23,3$ \\
\hline
\end{tabular}

Примечание: за 100\% принимали массу тела до операции, число меньше 100\% - отрицательная динамика веса, больше $100 \%$ - прибавка.

Таблица 3. Послеоперационные осложнения отдаленного периода, абс./\%

\begin{tabular}{|c|c|c|}
\hline \multirow[b]{2}{*}{ Временные параметры } & \multicolumn{2}{|l|}{ Проксимальная резекция желудка } \\
\hline & $\begin{array}{l}\text { Гастропластика в модификации } \\
\text { Meredino-Dillard } \\
\text { (I-я группа, n=44) }\end{array}$ & $\begin{array}{l}\text { Прямой пищеводно-желудочный } \\
\text { анастомоз } \\
\text { (II-я группа, n=42) }\end{array}$ \\
\hline Рефлюкс-эзофагит & $2 / 4,5$ & $6 / 14,3$ \\
\hline $\begin{array}{l}\text { Стриктура или рубцовый стеноз зоны } \\
\text { анастомоза }\end{array}$ & - & $1 / 2,4$ \\
\hline Демпинг - синдром & $1 / 2,3$ & $2 / 4,8$ \\
\hline $\begin{array}{l}\text { Эрозивно-язвенный анастомозит } \\
\text { пищеводно-кишечного соустья }\end{array}$ & $2 / 4,5$ & - \\
\hline $\begin{array}{l}\text { Эрозивно-язвенный анастомозит } \\
\text { пищеводно-желудочного соустья }\end{array}$ & - & $4 / 9,5$ \\
\hline Всего & $5 / 11,3$ & $13 / 31,0$ \\
\hline
\end{tabular}

Таблица 4. Отдаленные результаты операций шкале Visick,\%

\begin{tabular}{|l|l|l|}
\multirow{2}{*}{ Результат (самочувствие) } & Проксимальная резекция желудка \\
\cline { 2 - 3 } & $\begin{array}{l}\text { Гастропластика в модификации } \\
\text { Merendino-Dillard } \\
\text { (I-я группа, } \mathrm{n=44)}\end{array}$ & $\begin{array}{l}\text { Прямой пищеводно-желудочный } \\
\text { анастомоз (II-я группа, n=42) }\end{array}$ \\
\hline Хорошее & 72,7 & 47,8 \\
\hline Удовлетворительное & 19,2 & 34,3 \\
\hline Плохое & 8,1 & 17,3 \\
\hline
\end{tabular}

Для объективной оценки функциональных исходов различных методов реконструкции, проанализированы изменения питательного статуса и веса в сравнении с весом при выписке из стационара (табл. 2).

Следует отметить, что через 2 года и более в І-й группе более 6 раз сутки питались 5,9\% пациентов, тогда как во II-й 23,3\% (p<0,05). При этом 67,6\% пациентов І-й группы и 36,7\% II группы вернулись к прежнему (как до болезни) режиму питания - 3-4 раза в сутки $(\mathrm{p}<0,05)$.

При изучении динамики массы тела после 24 месяцев во II-й группе средний вес пациентов так же не пре-

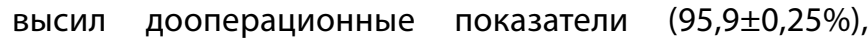
В то время как в І-й отмечено увеличение среднего

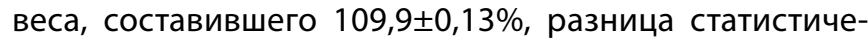
ски достоверна по отношению ко II-й группе $(p<0,05)$. 
Послеоперационные осложнения отдаленного периода наблюдались у 18 (20,9\%) пациентов (табл. 3).

При этом поздние осложнения, связанные с реконструкцией ЖКТ, были значительно выше при прямом пищеводно-желудочном анастомозе - 31,0\% против 11,3\% при реконструкции юногастропластикой в модификации Merendino-Dillard ( $p<0,05)$.

Как известно, ПРЖ сопровождается разрушением замыкательного механизма кардиоэзофагеальной зоны, что неизбежно приводит к развитию рефлюкс-эзофагита. В наших исследованиях частота рефлюкс-эзофагита наблюдалась в 8 (9,3\%) случаях: в І-й группе в $2(4,5 \%)$ и во II-й — в $6(14,3 \%)(p<0,05)$.

При ЭГДС эзофагит степени А (в соответствии с Лос-Анджелесской классификацией) чаще встречался в группе пациентов с гастропластикой в модификации Merendino-Dillard, а эзофагит более тяжёлых степеней (B и C) - в группе с «прямым» пищеводно-желудочным анастомозом $(p<0,01)$.

Следующим по частоте и важности осложнением отдаленного периода являлся эрозивно-язвенный анастомозит, выявленный у 6 (7,0\%) пациентов. При этом эрозивно-язвенный анастомозит пищеводно-кишечного соустья установлен у 2 (4,5\%) пациентов I группы, а эрозивно-язвенный анастомозит пищеводно-желудочного соустья (II-я группа) - у 4 (9,5\%). Ни в одном из осложнений отдалённого периода не потребовалось хирургической коррекции, все они были ликвидированы консервативными мероприятиями. Средняя длительность консервативного лечения составляла 9,9ะ1,2 дней.

Отдаленный результат операций оценивали и по трёхбалльной модифицированной шкале Visick, в которой учитываются самочувствие пациентов, ди- намика их питательного статуса, наличие тех или иных пищеварительных расстройств (табл. 4), при этом получена статистически достоверная разница в показателях хорошего самочувствия в сравниваемых группах: с гастропластикой в модификации Merendino-Dillard (72,7\%) и «прямого» пищеводно-желудочного анастомоза $(47,8 \%)(p<0,01)$.

Полученные результаты дают нам основание говорить о том, что после ПРЖ с гастропластикой в модификации Meredino-Dillard, отдаленный послеоперационный период протекает гораздо благоприятнее, чем после ПРЖ с наложением прямого пищеводно-желудочного анастомоза.

\section{Зак^ючение}

Проведенные исследования показали, что еюногастропластика в модифицированном варианте операции Merendino-Dillard,

в сравнении с прямым эзофагогастроанастомозом после проксимальной резекции желудка, практически не имеет противопоказаний, является безопасной хирургической методикой, не увеличивает кровопотерю и не сопровождается в ближайшем послеоперационном периоде ростом частоты хирургических осложнений и летальности.

В отдалённом периоде еюногастропластика (модификация операции Merendino-Dillard) приводит к существенно меньшим нарушениями работы пищеварительной системы (в 12,0\% случаев), по сравнению с эзофаго-гастростомией (в 30,0\%), значительному снижению (в 3,2 раза) послеоперационного рефлюкс-эзофагита и может быть рекомендована в качестве первичного способа реконструкции после субтотальной проксимальной резекции желудка.

\section{ЛИТЕРАТУРА}

1. Черноусов А.Ф., Хоробрых Т.В., Богопольский П.М. Хирургия язвенной болезни желудка и двенадцатиперстной кишки. М.: Практическая медицина. $2016 ; 352$ c.

2. Стилиди И.С. Неред С.Н. Современные представления об основных принципах хирургического лечения местно-распространенного рака желудка. Практическая онкология. 2009; 10(1): 20-27.

3. Каприн А.Д., Рухадзе Г.О., Костюк И.П., Шинкаркина А.П., Мозеров С.А., Двинских Н.Ю. Случай лечения гигантской гастроинтестинальной стромальной опухоли желудка с метастазом в серозной оболочке тонкой кишки. Онкология. Журнал им. П.А. Герцена. 2017a; 6(2): 45-50. doi: 10.17116/ onkolog20176245-50

4. Каприн А.Д., Сулейманов Э.А., Филоненко Е.В., Хомяков В.М., Урлова А.Н. Паллиативная хирургия в сочетании с интраоперационной фотодинамической терапией у больных раком желудка. Паллиативная медицина и реабилитация. 2017; 4: 30-31.

5. Черноусов А., Хоробрых Т., Зубарева М., Вычужанин Д., Горбунов А., Абдулхакимов Н., Уддин Л., Хоробрых В., Гельмутдинова Э. Актуальные проблемы хирургического лечения постгастрорезекционных синдромов. Врач. 2019; 6: 3-9. doi: 10.29296/25877305-2019-06-01

6. Fujitani K., Yang H.K., Mizusawa J., Kim Y.W., Terashima M., Han S.U., Iwasaki Y., Hyung W.J., Takagane A., Park D.J., Yoshikawa T., Hahn S., Nakamura K., Park C.H., Kurokawa Y., Bang Y.J., Park B.J., Sasako M., Tsujinaka T; REGATTA study investigators. Gastrectomy plus chemotherapy versus chemotherapy alone 
for advanced gastric cancer with a single non-curable factor (REGATTA): a phase 3, randomised controlled trial. Lancet Oncol. 2016 Mar; 17(3): 309-318. doi: 10.1016/S1470-2045(15)00553-7.

7. Fujitani K., Yang H.K., Mizusawa J., Kim Y.W., Terashima M., Han S.U., Iwasaki Y., Hyung W.J., Takagane A., Park D.J., Yoshikawa T., Hahn S., Nakamura K., Park C.H., Kurokawa Y., Bang Y.J., Park B.J., Sasako M., Tsujinaka T; REGATTA study investigators. Gastrectomy plus chemotherapy versus chemotherapy alone for advanced gastric cancer with a single non-curable factor (REGATTA): a phase 3, randomised controlled trial. Lancet Oncol. 2016 Mar; 17(3): 309-318. doi: 10.1016/S1470-2045(15)00553-7.

8. Максимов М.О., Тузиков С.А., Стрижаков Г.Н., Родионов Е.О. Результаты лечения проксимального рака желудка в зависимости от объема хирургического вмешательства. Сибирский онкологический журнал. 2017; 16 (5): 5-11. D0I: 10.21294/1814-4861-2017-16-5-5-11

9. Bang C.S., Park J.M., Baik G.H., Park J.J., Joo M.K., Jang J.Y., Jeon S.W., Choi S.C., Sung J.K., Cho K.B. Therapeutic Outcomes of Endoscopic Resection of Early Gastric Cancer with Undifferentiated-Type Histology: A Korean ESD Registry Database Analysis. Clin Endosc. 2017 Nov; 50(6):569-577. doi: 10.5946/ce.2017.017.

10. Haverkamp L., Seesing M.F., Ruurda J.P., Boone J., Hillegersberg R.V. Worldwide trends in surgical techniques in the treatment of esophageal and gastroesophageal junction cancer. Dis Esophagus. 2017 Jan 1; 30(1):1-7. doi: 10.1111/dote.12480.

11. Домрачев С.А., Израилов Р.Е., Кучер С.А. Минимально инвазивная хирургия при кардиоэзофагеальном раке. Онкология. Журнал им. П.А. Герцена. $2019 ; 1: 57-62$.

12. Израилов Р.Е., Поморцев Б.А., Васнев О.С., Кошкин М.А., Хатьков И.Е. Технические аспекты выполнения лапароскопической расширенной гастрэктомии у больных «истинным» кардиоэзофагеальным раком. Эндоскопическая хирургия. 2019; 25(3): 31-36. https://doi.org/10.17116/endoskop20192503127

13. Mizrahi M., Almogy G., Adar T., Lysy J. Dumping syndrome following nissen fundoplication in an adult patient diagnosed by continuous online $13 \mathrm{C} / 12 \mathrm{C}$ monitoring of C-Octanoic acid breath test "a case report". BMC Gastroenterol. 2011 Sep 19; 11: 98.

14. Корочанская Н.В., Генрих С.Р., Буложенко Н.А., Басенко М.А. Функциональные нарушения моторной активности тонкой кишки у пациентов С демпинг-синдромом. Вестник хирургической гастроэнтерологии. 2016; 6: 26-34.

15. Куртсеитов Н.Э., Дамбаев Г.Ц., Кошель А.П. Оригинальная тактика ведения больных с болезнью оперированного желудка. Вопросы реконструктивной и пластической хирургии. 2017; 1(60): 57-62. doi: 10.17223/1814147/60/08

16. Диомидова В.Н., Виноградова В.С. Ультразвуковая диагностика патологии оперированного желудка. Практическая медицина. $2018 ; 1(112): 86-89$.

17. Merendino K.A., Dillard D.H. The concept of sphincter substitution by an interposed jejunal segment for anatomic and physiologic abnormalities at the esophagogastric junction; with special reference to reflux esophagitis, cardiospasm and esophageal varices. Ann Surg. 1955 Sep; 142(3): 486-506. doi: 10.1097/00000658-195509000-00015.

( ) Тишакова Виктория Эдуардовна ( tishakova21@gmail.com ),Ручкин Дмитрий Валерьевич ( ruchkindmitry@gmail.com ),

Грицкевич Александр Анатольевич (vishnevskogo@ixv.ru), Ефименко Николай Алексеевич ( chiefsurgeon@mail.ru ).

Журнал «Современная наука: актуальные проблемы теории и практики» 